\title{
Insatisfação corporal de escolares e sua relação com o estado nutricional real
}

\author{
Miria Suzana Burgos ${ }^{1}$ \\ Luciana Tornquist ${ }^{2}$ \\ Debora Tornquist ${ }^{2}$ \\ Edna Linhares Garcia ${ }^{1}$ \\ Cézane Priscila Reuter ${ }^{1}$ \\ ${ }^{1}$ Universidade de Santa Cruz do Sul, RS, Brasil \\ ${ }^{2}$ Universidade Federal de Pelotas, RS, Brasil
}

\section{Resumo}

Este estudo objetiva identificar a prevalência da insatisfação corporal entre os escolares e relacioná-la com o seu real estado nutricional. Foram avaliados 1578 escolares do município de Santa Cruz do Sul-RS, através das medidas de peso e altura, para o cálculo do índice de massa corporal (IMC) e questionário para avaliar a satisfação com o peso corporal. Os resultados demonstram que a insatisfação corporal foi significativamente maior entre as meninas, os adolescentes e os níveis socioeconômicos mais altos. Os escolares, em sua maioria, mostraram uma percepção corporal condizente com o estado nutricional real, porém, alguns resultados preocupantes foram encontrados que apontam uma distorção entre a percepção corporal e o estado nutricional real, como o percentual de meninas eutróficas que gostariam de diminuir de peso, e escolares com excesso de peso que declararam estarem satisfeitos com seu peso ou ainda, que gostariam de aumentá-lo.

Palavras-chave: Imagem corporal; Índice de massa corporal; Crianças; Adolescente; Escolares.

\section{Body dissatisfaction among school children and its relation with the real nutritional state}

\begin{abstract}
This paper aims to identify the prevalence of body dissatisfaction among schoolchildren and relate it to their real nutritional status. The evaluation included 1578 schoolchildren in the municipality of Santa Cruz do Sul-RS, through the measures of weight and height, for the calculation of the body mass index (BMI) and questionnaire to evaluate the satisfaction with the body weight. The results show that body dissatisfaction was significantly higher among the girls, among the adolescents and among the higher economic classes. Most schoolchildren showed a body perception consistent with the actual nutritional status, however, some worrying results were found that point out a distortion between body perception and actual nutritional status, as the percentage of eutrophic girls who would like to decrease weight, and overweight school children who said they were satisfied with their weight or who would like to increase it.
\end{abstract}

Abstract

Keywords: Body image; Body mass index; Child; Adolescent; Schoolchildren.

\section{Insatisfacción corporal de estudiantes y su relación con el estado nutricional real}

\section{Resumen}

Este estudio tiene como objetivo identificar la prevalencia de la insatisfacción corporal entre los estudiantes y relacionarlo con su estado nutricional real. Evaluaron en 1578 escolares de la ciudad de Santa Cruz do Sul-RS, a través de las medidas de peso y altura, para el cálculo del índice de masa corporal (IMC) y el cuestionario para evaluar la satisfacción con el peso corporal. Los resultados muestran que la insatisfacción corporal fue significativamente mayor entre las niñas, los adolescentes y entre los más altos niveles socioeconómicos. Los escolares, en su mayoría, mostraron una percepción corporal acorde con el estado nutricional real, sin embargo, algunos resultados preocupantes fueron encontrados que apunta una distorsión entre la percepción corporal y el estado nutricional real, como el porcentaje de niñas eutróficas que les gustaría disminuir peso, y escolares con sobrepeso que declararon estar satisfechos con su peso o aún, que les gustaría aumentarlo.

Palabras clave: Imagen Corporal; Índice de Masa Corporal; Niño; Adolescente; Estudiantes. 


\section{Introdução}

A imagem corporal não é tida apenas como um fenômeno perceptivo. É um aspecto multidimensional que abrange componentes perceptivos, afetivos e de atitudes, estando ligada à autoestima, autoconfiança e a estabilidade emocional do sujeito (Huang, Norman, Zabinski, Calfas, \& Patrick, 2007). Desse modo, a imagem corporal está continuamente em modificação, em decorrência dos nossos encontros e desencontros, da intenção que estes implicam, seja amorosidade, hostilidade ou indiferença. Assim, as modificações que vão se produzindo na imagem corporal trazem sempre as marcas de nossa história, de cada um dos momentos de vida e todas essas transformações afetam diretamente a percepção que temos do nosso peso, tamanho, articulação, expansão, etc (Seixas, 2009).

A forma como percebemos nosso corpo também sofre influência dos ideais de corpo presentes na mídia, que na sociedade atual, exaltam como padrões de beleza um corpo magro (Larini \& Simões, 2009; Witt \& Schneider, 2011). Para Tabak, Mazur, Oblacińska, \& Jodkowska (2007) a introdução de um modelo de beleza, que para a maioria das pessoas é inatingível, leva a um afastamento da imagem corporal real da imagem desejada, o que pode ter por consequência sentimentos negativos, como a baixa autoestima, afetando de modo negativo a saúde e a qualidade de vida. $\mathrm{Na}$ atualidade, testemunhamos processos de sofrimento e de adoecimento produzidos na busca de atingir os ideais estéticos. Ao fugir do ideal estético-cultural, o corpo é tomado como anormal e/ou patológico. Acompanhar este ideal tem sido um dos processos mais conflitantes para os sujeitos, tendo em vista que os corpos desenhados pela mídia, pelos editoriais de moda, pelos reclames das academias mostram, como assinala Seixas (2009), que estamos sempre aquém do esperado, evidenciando um permanente déficit corporal.

A insatisfação corporal, em maior grau, pode afetar aspectos como autoestima, comportamento alimentar e desempenhos físico, cognitivo e psicossocial, prejudicando o desenvolvimento e a saúde do indivíduo como um todo (Larini \& Simões, 2009; Cardoso \& Velozo, 2009). Deste modo, uma percepção correta do próprio corpo pelas crianças e adolescentes, ou até mesmo da família, pode ser fator determinante para a procura de auxílio profissional e de tratamento adequado, em relação ao excesso de peso e também nos casos de superestimação do peso corporal, visto que estudos apontam relação entre imagem corporal e fatores de risco ligados à alimentação (Boa-Sorte et al., 2007; Nunes, Olinto, Barros, \& Camey, 2001; Stice, Hayward, Cameron, Killen, \& Taylor, 2000). Conforme Pinheiro e Jiménez (2010), a percepção do corpo distorcida e a insatisfação com o mesmo são critérios diagnósticos para transtornos alimentares. A prevalência destes transtornos tem aumentado e atingindo crianças em idades cada vez menores.

Diante do exposto, torna-se importante investigarmos a visão da imagem corporal que os indivíduos vêm formando sobre si, suas insatisfações com o peso corporal, e se estas insatisfações condizem com seu real estado nutricional. Nesta perspectiva, o presente estudo buscou identificar a prevalência de insatisfação corporal entre os escolares do município de Santa Cruz do Sul-RS e relacioná-la com o estado nutricional real da criança ou adolescente, por meio do índice de massa corporal - IMC.

\section{Metódo}

O presente estudo foi desenvolvido a partir do projeto de pesquisa "Saúde e estilo de vida na escola e na família: indicadores de saúde de escolares e sua relação com a saúde familiar, no meio urbano e rural de Santa Cruz do Sul", que foi realizado com crianças e adolescentes de 7 a 17 anos, alunos de escolas do centro, periferia e zona rural do município de Santa Cruz do Sul-RS.

A amostra final do estudo foi de 1578 escolares, escolhidos aleatoriamente de uma amostra estratificada por conglomerados (centro e norte, sul, leste e oeste da periferia da zona urbana e norte, sul, leste e oeste da zona rural), pertencentes a 18 escolas, sendo 14 da zona urbana e 4 da zona rural. Todos os pais ou responsáveis assinaram o termo de consentimento livre e esclarecido, autorizando a participação do escolar no estudo. A pesquisa tem aprovação do Comitê de Ética em Pesquisa com Seres Humanos da Universidade de Santa Cruz do Sul (CEP - UNISC - processo 4913/07).

Para avaliação da composição corporal da criança e adolescente, foi realizada avaliação antropométrica de peso e estatura (medida direta) e após, o cálculo do IMC (peso/altura ${ }^{2}$ ). A classificação do IMC foi realizada de acordo com as curvas de percentis do Centers for Disease Control and Prevention/National Center for Health Statistics (2000), de acordo com sexo e idade, considerando baixo peso $(<\mathrm{p} 5)$, peso normal $(\geq \mathrm{p} 5$ e $<\mathrm{p} 85)$, sobrepeso $(\mathrm{p} \geq 85 \mathrm{e}<\mathrm{p} 95)$ e obesidade ( $\geq$ p95). Para avaliar a satisfação com o peso corporal dos escolares foi utilizado o questionário Estilo de Vida, Saúde e Bem-estar, adaptado de Barros e Nahas (2003). Foram consideradas crianças, os escolares com até doze anos de idade incompletos, e adolescentes aqueles a partir de 12 anos, conforme o estatuto da criança e adolescente (Brasil, 2012). O nível socioeconômico foi classificado conforme o critério ABEP(2012). 
TABELA 1

Caracterização dos sujeitos

\begin{tabular}{lc}
\hline Sexo & $836(53,0)$ \\
Masculino & $742(47,0)$ \\
Feminino & \\
Faixa etária & $680(43,1)$ \\
Criança & $898(56,9)$ \\
Adolescente & \\
Região de moradia & $443(28,1$ \\
Centro & $785(49,7)$ \\
Periferia & $350(22,2)$ \\
Rural & \\
Nível socioeconômico & $724(45,8)$ \\
A-B & $716(45,4)$ \\
C & $138(8,8)$ \\
D-E & \\
Âmbito escolar & $177(11,2)$ \\
Particular & $1401(88,8)$ \\
Pública & \\
IMC & $1154(73,1)$ \\
Baixo Peso/Normal & $304(19,3)$ \\
Sobrepeso & $120(7,6)$ \\
Obesidade &
\end{tabular}

Os dados foram analisados no programa estatístico SPSS for Windows 22.0 (IBM, Chicago, EUA), através de estatística descritiva (frequências e percentuais). Para avaliar as razões de prevalência da insatisfação corporal com os dados sociodemográficos e com o
IMC, foi utilizada a regressão de Poisson. Foram consideradas diferenças significativas para $\mathrm{p}<0,05$, através do teste de qui-quadrado.

Para relacionarmos o IMC dos avaliados com a insatisfação com o peso corporal, optamos por dividir os avaliados em dois grupos: grupo 1 e grupo 2. Sendo o grupo 1, os escolares com IMC classificado na faixa de baixo peso ou de peso normal e o grupo 2, escolares com excesso de peso (sobrepeso ou obesidade). Os escolares de baixo peso e peso normal foram incluídos no mesmo grupo, devido a prevalência de casos de baixo peso ser muito baixa entre os escolares, sendo de apenas 25 escolares (1,6\% da amostra total).

\section{Resultados}

Observa-se que a prevalência de insatisfação (desejo de aumentar ou diminuir de peso) foi significativamente maior entre o sexo feminino, entre os adolescentes e entre as classes econômicas mais altas. Entre as escolares do sexo feminino, 41,5\% declararam o desejo de diminuir seu peso. Entre os escolares adolescentes, 40,0\%. Em comparação entre as classes ecônomicas, $34,0 \%$ dos escolares das classes mais altas (A e B) desejam diminuir de peso, enquanto entre os escolares das classes mais baixas (C e D) este percentual é de $20,3 \%$. Já, em relação a região de moradia e o tipo de escola (pública ou particular) não foram observadas diferenças para a satisfação corporal dos escolares (Tabela 2).

TABELA 2

Satisfação com o peso corporal

\begin{tabular}{|c|c|c|c|c|}
\hline & Satisfeito & Não, gostaria de aumentar & Não, gostaria de diminuir & \multirow{2}{*}{$p$} \\
\hline & $n(\%)$ & $n(\%)$ & $n(\%)$ & \\
\hline \multicolumn{5}{|l|}{ Sexo } \\
\hline Masculino & $544(65,1)$ & $103(12,3)$ & $189(22,6)$ & \multirow[t]{2}{*}{0,000} \\
\hline Feminino & $379(51,1)$ & $55(7,4)$ & $308(41,5)$ & \\
\hline \multicolumn{5}{|l|}{ Faixa etária } \\
\hline 7-11 anos & $497(73,1)$ & $45(6,6)$ & $138(20,3)$ & \multirow[t]{2}{*}{0,000} \\
\hline 12-17 anos & $426(47,4)$ & $113(12,6)$ & $359(40,0)$ & \\
\hline \multicolumn{5}{|l|}{ Região } \\
\hline Centro & $243(54,9)$ & $49(11,1)$ & $151(34,0)$ & \multirow[t]{3}{*}{0,487} \\
\hline Periferia & $469(59,7)$ & $75(9,6)$ & $241(30,7)$ & \\
\hline Rural & $211(60,3)$ & $34(9,7)$ & $105(30,0)$ & \\
\hline \multicolumn{5}{|c|}{ Nível socioeconômico } \\
\hline A-B & $404(55,8)$ & $70(9,7)$ & $250(34,5)$ & \multirow[t]{3}{*}{0,021} \\
\hline $\mathrm{C}$ & $424(59,2)$ & $73(10,2)$ & $219(30,6)$ & \\
\hline D-E & $95(68,8)$ & $15(10,9)$ & $28(20,3)$ & \\
\hline \multicolumn{5}{|l|}{ Âmbito escolar } \\
\hline Particular & $101(57,1)$ & $16(9,0)$ & $60(33,9)$ & \multirow[t]{3}{*}{0,732} \\
\hline Pública & $822(58,7)$ & $142(10,1)$ & $437(31,2)$ & \\
\hline Total & $923(58,5)$ & $158(10,0)$ & $497(31,5)$ & \\
\hline
\end{tabular}


A insatisfação com o peso corporal foi significativamente maior para os escolares do grupo 2 - sobrepeso/obesidade (Tabela 3), com maior prevalência do desejo de diminuir de peso, em ambos os sexos. Entre os sujeitos do grupo 1 - baixo peso/ peso normal, podemos perceber que, para o sexo masculino, predominou o desejo de aumentar o peso corporal, enquanto para o sexo feminino, destaca-se o percentual de escolares que gostaria de diminuir seu peso $(33,4 \%)$.

De acordo com a Tabela 4, observa-se maior prevalência de insatisfação corporal entre os escolares do sexo feminino (RP: 1,$16 ; \mathrm{p}<0,001$ ), entre os adolescentes (RP: 1,$19 ; \mathrm{p}<0,001)$ e entre os escolares com sobrepeso e obesidade (RP: 1,$34 ; p<0,001$ ).

TABELA 3

Relação do IMC com satisfação com o peso corporal

\begin{tabular}{|c|c|c|c|c|c|c|}
\hline & \multicolumn{3}{|c|}{ Masculino } & \multicolumn{3}{|c|}{ Feminino } \\
\hline & $\begin{array}{c}\text { Grupo } 1 \\
N=609\end{array}$ & $\begin{array}{c}\text { Grupo } 2 \\
N=227\end{array}$ & \multirow[t]{2}{*}{$p$} & $\begin{array}{c}\text { Grupo } 1 \\
N=545\end{array}$ & $\begin{array}{c}\text { Grupo } 2 \\
N=197\end{array}$ & \multirow[t]{2}{*}{$p$} \\
\hline & $n(\%)$ & $n(\%)$ & & $n(\%)$ & $n(\%)$ & \\
\hline \multicolumn{7}{|l|}{ Satisfação com o peso } \\
\hline Satisfeito & $450(73,9)$ & $94(41,4)$ & 0,000 & $320(58,7)$ & $59(29,9)$ & 0,000 \\
\hline Não, gostaria de aumentar & $95(15,6)$ & $8(3,5)$ & & $43(7,9)$ & $12(6,1)$ & \\
\hline Não, gostaria de diminuir & $64(10,5)$ & $125(55,1)$ & & $182(33,4)$ & $126(64,0)$ & \\
\hline
\end{tabular}

Grupo 1: baixo peso/normal; Grupo 2: sobrepeso/obesidade.

\section{TABELA 4}

Razões de prevalência para as características sociodemográficas para o índice de massa corporal, considerando a insatisfação corporal e percepção de saúde

\begin{tabular}{|c|c|c|}
\hline & \multicolumn{2}{|c|}{ Insatisfação corporal* } \\
\hline & $R P(I C 95 \%)$ & $p$ \\
\hline \multicolumn{3}{|l|}{ Sexo } \\
\hline Masculino & 1 & \\
\hline Feminino & $1,16(1,12-1,19)$ & $<0,001$ \\
\hline \multicolumn{3}{|l|}{ Faixa etária } \\
\hline Criança & 1 & \\
\hline Adolescente & $1,19(1,16-1,23)$ & $<0,001$ \\
\hline \multicolumn{3}{|c|}{ Indicadores socioeconômicos } \\
\hline A-B & 1 & \\
\hline $\mathrm{C}$ & $0,99(0,95-1,02)$ & 0,531 \\
\hline D-E & $0,92(0,87-0,97)$ & 0,004 \\
\hline \multicolumn{3}{|l|}{ Região de moradia } \\
\hline Centro & 1 & \\
\hline Periferia & $0,98(0,94-1,03)$ & 0,410 \\
\hline Rural & $0,97(0,92-1,02)$ & 0,177 \\
\hline \multicolumn{3}{|l|}{ Âmbito escolar } \\
\hline Particular & 1 & \\
\hline Pública & $0,99(0,93-1,05)$ & 0,700 \\
\hline \multicolumn{3}{|l|}{ IMC } \\
\hline Baixo peso/Normal & 1 & \\
\hline Sobrepeso/Obesidade & $1,34(1,30-1,39)$ & $<0,001$ \\
\hline
\end{tabular}

* Insatisfação corporal pelo excesso de peso; percepção de saúde regular/ruim. IMC: índice de massa corporal; RP: razão de prevalência; IC: intervalo de confiança.

\section{Discussão}

Nos resultados de nosso estudo, encontramos uma maior insatisfação com o corpo entre os escolares do sexo feminino. Estudos anteriores, realizados com escolares no Rio Grande do Sul (Triches \& Giugliani, 2007; Toni et al., 2012), Santa Catarina (Adami, Frainer, Santos, Fernandes, \& Oliveira, 2008; Matias, Rolim, Kretzer, Schmoelz, \& Andrade, 2010; Petroski, Pelegrini, \& Glaner, 2012) e Estados Unidos (Jones, Vigfusdottir, \& Lee, 2004) também observaram maior prevalência de insatisfação corporal entre o sexo feminino. De contrário modo, outros estudos encontram maior prevalência de insatisfação corporal entre os indivíduos do sexo masculino (Fidelix, Silva, Pelegrini, Silva, \& Petroski, 2011; Pereira, Graup, Lopes, Borgatto, \& Daronco, 2009; Santini \& Kirsten, 2012; Pelegrini, Silva, Silva, \& Petroski, 2011; Leite, Ferrazzi, Mezadri, \& Höfelmann, 2014) e estudos com adolescentes do oeste de Santa Catarina (SC) e Norte do Rio Grande do Sul (Petroski, Pelegrini, \& Glaner, 2009) e de Porto Alegre (Pinheiro \& Giugliani, 2006) não encontraram diferenças na insatisfação corporal entre os sexos.

Para Alves, Pinto, Alves, Mota \& Leirós (2009) o fato de a insatisfação corporal estar presente em ambos os sexos está ligado ao não enquadramento nos padrões estético-culturais da sociedade atual. Pereira et al. (2009), encontrou uma tendência das crianças e 
adolescentes do sexo masculino desejarem corpos mais fortes, e entre o sexo feminino, corpos mais magros. Semelhantemente, estudo realizado com adolescentes de Santa Catarina, constatou que a insatisfação corporal estava associada com o IMC baixo e a obesidade abdominal, para o sexo masculino e com o IMC e percentual de gordura elevados, para o sexo feminino (Glaner, Pelegrini, Cordoba, \& Pozzobon, 2013). Também em estudos de Petroski et al. (2012), Santini e Kirsten(2012) e Dumith et al. (2012), as meninas estão mais insatisfeitas com o excesso de peso e os meninos com a magreza. Resultados semelhantes também foram encontrados em nosso estudo.

Fleitlich, Larino, Cobelo e Cordás (2000) afirmam que mesmo quando os adolescentes se encontram com o peso adequado ou até abaixo dele, costumam se sentir gordos ou desproporcionais, o que os autores denominam de distorção da imagem corporal. Este fato é percebido em nosso estudo, onde se constatou maior insatisfação corporal entre os adolescentes (12 a 17 anos), e um relevante percentual de escolares do grupo de peso normal e de baixo peso, mostrando-se insatisfeitos com seu peso.

Esta adequação da autopercepção do corpo e da insatisfação com o mesmo, em relação ao real estado nutricional é demarcado pelos padrões de beleza estabelecidos e divulgados pela mídia e pela necessidade de aceitação que os jovens têm no seu grupo de convivência (Graup et al., 2008). Estudo em Minas Gerais (Fortes, Amaral, Almeida, \& Ferreira, 2013) demonstrou esta relação, onde os resultados evidenciaram que, em adolescentes do sexo femino, a internalização do ideal de magreza e de corpo atlético modularam os níveis de insatisfação corporal com o peso e com a aparência física

Corroborando com nossos achados, Triches e Giugliani (2007) em seu estudo em duas cidades do Rio Grande do Sul, perceberam uma tendência, embora não significativa, do desejo de emagrecer, aumentar paralelamente ao aumento da idade e o de engordar, diminuir. Já, estudos na cidade de São Paulo - SP (Branco, Hilário, \& Cintra, 2006), em Porto AlegreRS (Pinheiro \& Giugliani, 2006) e em Januária-MG (Fidelix et al., 2011), não encontraram diferenças significativas entre as faixas etárias. Em estudo em São Luiz-MA não foram encontradas diferenças significativas para insatisfação corporal segundo a idade e o gênero (Pinheiro \& Jiménez, 2010).

Com relação à região em que moram os escolares, não houve diferenças significativas, entretanto, percebeu-se que ocorre maior satisfação com o peso corporal entre os escolares da zona rural, sendo os escolares da zona central, os mais insatisfeitos.
Corroborando com nossos achados, Triches e Giugliani (2007), Petroski et al. (2012) e Duca et al. (2010) verificaram em seus estudos maiores índices de insatisfação corporal na zona urbana. Já Petroski et al. (2009) encontraram índices de insatisfação corporal semelhantes entre as áreas domiciliares $(64,2 \%$ zona rural e 62,8\% urbana). Também Welch, Gross, Bronner, Dewberry-Moore e Paige (2004), Fidelix et al. (2011) e Ferrazzi et al. (2014) não observaram associação entre imagem corporal e região geográfica.

Quanto ao nível socioeconômico, percebeu-se que os escolares de classes mais baixas (D e E) estão mais satisfeitos com seu corpo. Ogden e Stewart (2000) relataram em seu estudo, associação entre classe social e preocupações com o peso, tendo as meninas das classes mais altas, relatado maior prevalência de insatisfação corporal e maior distorção da imagem corporal. Palma et al. (2013) também encontraram maior insatisfação entre as adolescentes de classes mais altas, em seu estudo no Rio de Janeiro. De modo contrário, Pereira et al. (2009) encontraram maior prevalência de insatisfação corporal entre as classes econômicas mais baixas. Também, estudo com crianças e adolescentes da Austrália (O'dea, 2008), encontrou maior insatisfação entre os escolares de classes mais baixas.

Com relação ao âmbito escolar, percebeu-se, em nosso estudo, níveis de satisfação corporal bem semelhantes entre os escolares da rede particular e privada. O mesmo não foi encontrado em estudo realizado em Belo Horizonte (Beling et al., 2012), onde as adolescentes das escolas particulares apresentavam 1,87 vezes mais chance de apresentar insatisfação com o corpo. Já estudo com escolares de Porto Alegre (Pinheiro \& Giugliani, 2006) encontrou associação entre estudar em escola pública e insatisfação corporal.

Os achados do atual estudo indicam que os escolares com sobrepeso ou obesidade foram os mais insatisfeitos, com predomínio do desejo de diminuir seu peso corporal, em ambos os sexos $(55,1 \%$ masculino e $64,0 \%$ feminino), demonstrando uma boa percepção corporal entre a maioria dos escolares deste grupo. Entre os escolares com baixo peso ou com peso adequado, também foi encontrada uma percepção corporal adequada entre a maioria dos avaliados, especialmente entre o sexo masculino, visto que estes declaram estarem satisfeitos com seu peso atual $(73,9 \%$ masculino e 58,7\% feminino). Entretanto, entre este grupo, destaca-se também o percentual de escolares que gostaria de diminuir seu peso, para o sexo feminino $(33,4 \%)$, ou aumentar, para o masculino $(15,6 \%)$.

Corroborando com nossos achados, estudo com escolares de Florianópolis-SC (Graup et al., 2008), 
apontou que entre os escolares insatisfeitos, o maior percentual também foi de escolares que desejam perder peso, e este percentual também foi mais elevado para o sexo feminino. Já, Erickson, Robinson, Haydel e Killen (2000), ao avaliarem escolares do norte da Califórnia (EUA), observaram maior preocupação com excesso de peso, entre o sexo masculino. Em estudo realizado com escolares do Paraná (Cardoso \& Velozo, 2009), $65 \%$ das alunas relataram que se sentiriam mais bonitas se fossem mais magras. Também, em estudo realizado com escolares de Três de Maio-RS (Corseuil, Pelegrini, Beck, \& Petroski, 2009) e outro realizado no interior de Minas Gerais (Vilela, Lamounier, Dellaretti-Filho, Barros-Neto, \& Horta, 2004), foi maior o desejo de diminuir o peso corporal $(71,7 \%$ no $\mathrm{RS}$ e $52 \%$ em $\mathrm{MG})$ do que de aumentá-lo. Porém, outro estudo em Minas Gerais observou maior prevalência do desejo em aumentar o tamanho corporal, em ambos os sexos (Fidelix et al., 2011).

Em estudo, avaliando escolares de Porto AlegreRS (Pinheiro \& Giugliani, 2006), observou-se que 76\% dos escolares, classificados dentro da zona normal de IMC, relataram insatisfação com o corpo, sendo que $43 \%$ desejavam diminuir seu peso e $33 \%$, ter um corpo maior. Já em estudo realizado em Macapá - AP (Santos, Monteiro, Silva, Sousa, \& Novaes, 2009), a maior prevalência de insatisfação corporal ocorreu entre os escolares que apresentavam baixo peso, seguido pelos escolares eutróficos (peso normal). Outro estudo em Porto Alegre-RS, meninas que apresentavam baixo IMC não desejavam emagrecer, estando divididas entre o desejo de manter ou aumentar seu peso. Já, 93,8\% das alunas com excesso de peso, e $85,7 \%$ com obesidade demonstraram vontade de diminuir de peso. Nas meninas que apresentavam eutrofia, 34,78\% estavam satisfeitas com seu peso, 26,09\% declararam o desejo de engordar e 39,13\% gostariam de diminuir seu peso (Boklis, Pellanda, Gama, \& Stenzel, 2013).

Em estudo com escolares de Portugal (Fonseca \& Matos, 2005), observou-se boa percepção corporal do grupo classificado com excesso de peso, onde $65,5 \%$ deste grupo relataram perceber seu corpo com sobrepeso. Porém, cabe destacar em nosso estudo, o percentual de escolares que declararam estar satisfeitos com seu peso, mesmo apresentado excesso de peso. De mesmo modo, estudo com alunos de escolas públicas de Santa Catarina (Duca et al., 2010), observou que entre os alunos com excesso de peso, 20,9\% diziam-se satisfeitos com seu peso corporal.

De maneira geral, percebe-se que maioria dos escolares avaliados, apresenta uma correta percepção corporal, independente da classificação do IMC. Indo ao encontro de nossos achados, estudo na cidade de
Salvador-BA (Boa-Sorte et al., 2007), observou que $64,8 \%$ dos escolares tinham percepção correta de seu peso, com relação à classificação do IMC, tendo $16,8 \%$ dos escolares superestimado seu peso e $18,4 \%$ subestimado. Entre os avaliados que estimavam possuir excesso de peso, $79,7 \%$ eram do sexo feminino e $57,9 \%$ não estavam acima do peso. Wiederman e Pryor (2000) alertam para o risco deste desejo de ficar ainda mais magra, motivado pela estética, se tornar um risco para a saúde, tendo estas uma maior tendência a doenças como a bulimia e a depressão.

Em estudo realizado em São Luiz-MA (Pinheiro \& Jiménez, 2010), as meninas apresentatam uma percepção corporal mais acurada, com uma perceção mais próxima a sua imagem verdadeira. Ainda, neste estudo, 16,8\% dos avaliados, de ambos os sexos, apresentam uma percepção correta de seu corpo, $16,8 \%$ percebem-se mais gordos e $66,4 \%$ percebem-se mais magros do que a realidade. $\mathrm{O}$ estudo ainda mostra que os sujeitos com sobrepeso apresentam maior distorção da percepção corporal que os demais avaliados. O nível de insatisfação corporal da população do estudo foi de $64 \%$, sendo que $39,8 \%$ dos participantes gostariam de diminuir de peso e $24,2 \%$ aumentar.

Também na cidade de São Paulo-SP (Branco et al., 2006), observou-se que 66,01\% dos escolares tinham percepção adequada de seu peso, $28,34 \%$ superestimavam e 5,65\% subestimavam, com percepção corporal mais errônea para o sexo feminino. Em estudo em Pelotas-RS (Dumith et al., 2012), entre os adolescentes classificados como magros, cerca de três quartos gostariam de aumentar de peso e entre os obesos, mais de $90 \%$ desejavam diminuir a silhueta. Em um estudo realizado nas cidades de Morro Reuter e Dois Irmãos (RS) observou-se que os escolares mais insatisfeitos foram os que apresentaram risco para desenvolver obesidade, escolares obesos ou com baixo peso. Ainda assim, entre os escolares classificados como eutróficos, a maioria estava insatisfeita com seu peso corporal (Triches \& Giugliani, 2007).

Em estudo realizado com adolescentes de Fiji, Tonga e Austrália (McCabe, Ricciardelli, Waqa, Goundar, \& Fotu, 2009), os adolescentes com excesso de peso também se mostraram mais insatisfeitos com seus corpos do que aqueles que estavam com peso normal. Igualmente, em estudo com adolescentes do Rio de Janeiro, as que apresentaram sobrepeso também demonstraram maior insatisfação com a massa corporal, quando comparadas às adolescentes com baixo peso ou peso normal, onde $42,48 \%$ adolescentes manifestaram insatisfação com o corpo, sendo que 30,45\% desejariam perder peso, enquanto $12,01 \%$ gostariam de ganhar (Palma et al., 2013). Também Petroski et al. (2009) 
observaram boa percepção corporal dos escolares em seus estudos, revelando que os adolescentes com baixo peso e excesso de peso apresentaram maior desejo em aumentar e reduzir seu peso, respectivamente.

Em estudo realizado na cidade de Três de Maio-RS, os resultados demonstraram que os escolares com IMC e percentual de gordura inadequado, demonstraram respectivamente 3,38 e 3,76 vezes mais chances de estarem insatisfeitos com seu corpo (Corseuil et al., 2009), corroborando com nossos achados. De modo semelhante, estudo com adolescentes de Minas Gerais (Beling et al., 2012) constatou que as adolescentes com sobrepeso possuíam quase 5 vezes mais chances de insatisfação, que as escolares com peso adequado, e para as obesas, essa chance aumentava para 6,85 . O mesmo também foi encontrado por Toni et al. (2012), onde escolares acima do peso apresentaram cinco vezes mais chances de estarem insatisfeitos com sua imagem corporal.

\section{Considerações finais}

Os achados do presente estudo permitem concluir que os escolares avaliados demonstraram, em sua maioria, uma percepção corporal adequada ao seu peso, mas resultados preocupantes foram encontrados, que demonstram uma distorção na percepção corporal com o seu real estado nutricional em uma parcela dos escolares avaliados, especialmente referentes às escolares do sexo feminino, que mesmo apresentando peso normal, gostariam de diminuir de peso, e também o percentual de escolares nas faixas de excesso de peso que declararam estarem satisfeitos com seu peso ou ainda que gostariam de aumentá-lo. Os resultados demonstram ainda que a insatisfação corporal foi significativamente maior entre as meninas, entre os adolescentes e entre os níveis econômicos mais elevados.

A distorção na percepção corporal entre crianças e adolescentes faz-se preocupante uma vez que em casos de superestimação do peso corporal há uma relação entre a percepção corporal e os fatores de risco ligados à alimentação, como transtornos alimentares. E, em relação aos escolares com excesso de peso, a percepção correta do próprio corpo pode ser fator determinante para a procura de auxílio profissional e de tratamento adequado à perda de peso. Além disso, os ideais de corpo apresentados pela mídia e idolatrados pela sociedade atual, que apresentam corpos magros e esbeltos, fazem com que, este padrão inatingível para muitas pessoas, levem a uma processo de baixa autoestima e adoecimento psíquico, afetando de forma negativa a qualidade de vida e a saúde.

Deste modo, destacamos a importância de orientações em saúde dentro do ambiente escolar, partindo de um trabalho interdisciplinar, especialmente no âmbito nutricional, da atividade física e da psicologia, visando desenvolver entre estes escolares, uma percepção saudável de seu próprio corpo, com a manutenção de um peso corporal adequado e a adoção de um estilo de vida saudável, para que também os índices de satisfação corporal e de saúde sejam satisfatórios.

\section{Referências}

Associação Brasileira de Empresas de Pesquisa. (2012). Critério de Classificação Econômica Brasil.

Adami, F., Frainer, D. E. S., Santos, J. S., Fernandes, T. C., \& Oliveira, F. R. (2008). Insatisfação corporal e atividade física em adolescentes da região continental de Florianópolis. Psicologia: Teoria e Pesquisa, 24(2), 143-149. https:// doi.org/10.1590/S0102-37722008000200003

Alves, D., Pinto, M., Alves, S., Mota, A., \& Leirós, V. (2009). Cultura e imagem corporal. Motricidade, 5(1), 1-20. https://doi.org/10.6063/motricidade.5(1).184

Barros, M. V. G. \& Nahas, M. V. (2003) Medidas da atividade física: teoria e aplicação em diversos grupos populacionais. Londrina: Midiograf.

Beling, M. T. C., Ferreira, M. F. R., Araújo, A. M. M., Barros, A. F. S., Beling, G., \& Lamounier, J. A. (2012). Alterações na imagem corporal entre adolescentes do sexo feminino e fatores associados. Adolescência \& Saúde, 9(4), 11-18.

Boa-sorte, N., Neri, L. A., Leite, M. E. Q., Brito, S. M., Meirelles, A. R., Luduvice, F. B., Santos, J. P., Viveiros, M. R., \& Ribeiro Júnior, H. C. (2007). Maternal perceptions and self-perception of the nutritional status of children and adolescents from private schools. Jornal de Pediatria, 83(4), 349-356. https://doi.org/10.2223/JPED.1678

Boklis, M., Pellanda, L. C., Gama, C. M., \& Stenzel, L. M. (2013). A Percepção de Meninas Sobre as Atitudes Maternas e sua Relação com a (In)satisfação Corporal. Psico (Porto Alegre), 44(4), 474-481.

Branco, L. M., Hilário, M. O. E., \& Cintra, I. P. (2006). Percepção e satisfação corporal em adolescentes e a relação com seu estado nutricional. Revista de Psiquiatria Clínica, 33(6), 292-296. https://doi.org/10.1590/S010160832006000600001 
Brasil. (2012). Estatuto da Criança e do adolescente. 7. ed. Brasília: Câmara dos Deputados.

Cardoso, E. S. \& Velozo, E. (2009). O corpo feminino na adolescência: os saberes de estudantes sobre anorexia e bulimia. Cinergis, 10(2), 62-68. http://dx.doi.org/10.17058/cinergis.v10i2.1716

Centers for Disease Control and Prevention/ National Center for Health Statistics. (2000). CDC Growth Charts: United States. Disponível em: http://www.cdc.gov/growthcharts

Corseuil, M. W., Pelegrini, A., Beck, C., \& Petroski, E. L. (2009). Prevalência de insatisfação com a imagem corporal e sua associação com a inadequação nutricional em adolescentes. Revista da Educação Física/UEM, 20(1), 25-31. http://dx.doi.org/10.4025/reveducfisv20n1p25-31

Duca, G. F. D., Garcia, L. M. T., Sousa, T. F., Oliveira, E. S. A, \& Nahas, M. V. (2010). Insatisfação com o peso corporal e fatores associados em adolescentes. Revista Paulista de Pediatria, 28(4), 340-346. https://doi.org/10.1590/S010305822010000400009

Dumith, S. C., Menezes, A. M. B., Bielemann, R. M., Petresco, S., Silva, I. C. M., Linhares, R. S., Amorim, T. C., Duarte, D. V.; Araújo, C. L. P., \& Santos, J. V. (2012). Insatisfação corporal em adolescentes: um estudo de base populacional. Ciência \& Saúde Coletiva, 17(9), 2499-2505. https://doi.org/10.1590/S1413-81232012000900030

Erickson, S. J., Robinson, T. N., Haydel, F., Joel, D., Killen, J. D. (2000). Are Overweight Children Unhappy? Body Mass Index, Depressive Symptoms, and Overweight Concerns in Elementary School Children. Archives of pediatrics \& adolescent medicine, 154(9), 931-935 https://doi.org/10.1001/archpedi.154.9.931

Fidelix, Y. L., Silva, D. A. S., Pelegrini, A., Silva, A. F., \& Petroski, E. L. (2011). Insatisfação com a imagem corporal em adolescentes de uma cidade de pequeno porte: associação com sexo, idade e zona de domicílio. Revista Brasileira de Cineantropometria \& Desempenho Humano, 13(3), 202-207. https://doi.org/10.5007/1980-0037.2011v13n3p202

Fleitlich, B. W., Larino, M. A., Cobelo, A., \& Cordás, T. A. (2000). Anorexia nervosa na adolescência. Jornal de Pediatria, 76(3), 323-329. https://doi.org/10.2223/JPED.170

Fonseca, H. \& Matos, M. G. (2005). Perception of overweight and obesity among Portuguese adolescents: an overview of associated factors. The European Journal of Public Health, 15(3), 323-328. https://doi.org/10.1093/eurpub/ cki071

Fortes, L. S., Amaral, A. C. S., Almeida, S. S., Ferreira, M. E. C. (2013). Internalização do Ideal de Magreza e Insatisfação com a Imagem Corporal em Meninas Adolescentes. Psico (Porto Alegre), 44(3), 432-438.

Glaner, M. F., Pelegrini, A., Cordoba, C. O., \& Pozzobon, M. E. (2013). Associação entre insatisfação com a imagem corporal e indicadores antropométricos em adolescentes. Revista Brasileira de Educação Física \& Esporte, 27(1), 129-36. https://doi.org/10.1590/S1807-55092013000100013

Graup, S., Pereira, E. F., Lopes, A. S., Araújo, V. C., Legnani, R. F. S., \& Borgatto, A. F. (2008). Associação entre a percepção da imagem corporal e indicadores antropométricos de escolares. Revista Brasileira de Educação Física \& Esporte, 22(2), 129-138. http://dx.doi.org/10.1590/S1807-55092008000 200004

Huang, J. S., Norman, G. J., Zabinski, M. F., Calfas, K., \& Patrick, K. (2007). Body image and self-steem among adolescent undergoing an intervention targeting dietary and physical activity behaviours. Journal of Adolescent Health, 40(3), 245-251. https://doi.org/10.1016/j.jadohealth.2006.09.026

Jones, D. C., Vigfusdottir, T. H., \& Lee, Y. (2004). Body Image and the appearance culture among adolescent girls and boys: an examination of friend conversations, peer criticism, appearance magazines, and the internalization of appearance ideals. Journal of Adolescent Research, 19, 323-339. https://doi.org/10.1177/0743558403258847

Larini, K. C. P., \& Simões, R. (2009). Sobrepeso ou obesidade: a visão de corpo de mulheres maduras. Movimento \& Percepção, 10(4), 67-85.

Leite, A. C. B., Ferrazzi, N. B., Mezadri, T., Höfelmann, D. A. (2014). Insatisfação corporal em escolares de uma cidade do sul do brasil. Journal of Human Growth and Development, 24(1), 54-61. https://doi.org/10.7322/jhgd.72154

Matias, T. S., Rolim, M. K. S. B., Kretzer, F. L., Schmoelz, C. P, \& Andrade, A. (2010). Satisfação corporal associada à prática de atividade física na adolescência. Motriz, 16(2), 370-378. https://doi.org/10.5016/19806574.2010v16n2p370

McCabe, M. P., Ricciardelli, L., Waqa, G., Goundar, R., Fotu, K. (2009). Body image and body change strategies among adolescent males and females from Fiji, Tonga and Australia. Body Image, 6(4), 299-303. https://doi.org/10.1016/j. bodyim. 2009.06.001

Nunes, M. A., Olinto, M. T. A., Barros, F. C., \& Camey, S. (2001). Influência da percepção do peso e do índice de massa corporal nos comportamentos alimentares anormais. Revista Brasileira de Psiquiatria, 23(1), 21-27. https:// doi.org/10.1590/S1516-44462001000100006

O'dea, J. A. (2008). Gender, ethnicity, culture and social class influences on childhood obesity among Australian schoolchildren: implications for treatment, prevention and community education. Health \& Social Care in the Community, 16(3), 282-290. https://doi.org/10.1111/j.1365-2524.2008. 00768.x

Ogden, J., \& Stewart, J. (2000). The role of the mother-daghter relationship in explaining weight concern. International Journal of Eating Disorders, 28, 78-83. https://doi.org/10.1002/(SICI)1098-108X(200007)28:1<78::AIDEAT9>3.0.CO;2-N

Palma, A., Resende, F., Marques, R. S., Assis, M., \& Teves, N. (2013). Insatisfação com o peso e a massa corporal em estudantes do ensino fundamental e médio do sexo feminino no município do Rio de janeiro. Revista Brasileira de Ciências e Esporte, 35(1), 51-64. https://doi.org/10.1590/S0101-32892013000100006 
Pelegrini, A., Silva, D. A. S., Silva, A. F., Petroski, E. L. (2011). Insatisfação corporal associada a indicadores antropométricos em adolescentes de uma cidade com índice de desenvolvimento humano médio a baixo. Revista Brasileira de Ciências e Esporte, 33(3), 687-698. http://dx.doi.org/10.1590/S0101-32892011000300011

Pereira, E. F., Graup, S., Lopes, A. S., Borgatto, A. F., Daronco, L. S. E. (2009). Percepção da imagem corporal de crianças e adolescentes com diferentes níveis socioeconômicos na cidade de Florianópolis, Santa Catarina, Brasil. Revista Brasileira de Saúde Materno Infantil, 9(3), 253-262. http://dx.doi.org/10.1590/S1519-38292009000300004

Petroski, E. L., Pelegrini, A., Glaner, M. F. (2012). Motivos e prevalência de insatisfação com a imagem corporal em adolescentes. Ciência \& Saúde Coletiva, 17(4), 1071-1077. https://doi.org/10.1590/S1413-81232012000400028

Petroski, E. L., Pelegrini, A., \& Glaner, M. F. (2009). Insatisfação corporal em adolescentes rurais e urbanos. Motricidade, 5(4), 13-25. https://doi.org/10.6063/motricidade.5(4).167

Pinheiro, A. P., Giugliani, E. R. J. (2006). Body dissatisfaction in Brazilian schoolchildren: prevalence and associated factors. Revista Saúde Pública, 40(3), 489-496. https://doi.org/10.1590/S0034-89102006000300018

Pinheiro, N. \& Jiménez, M. (2010). Percepção e insatisfação corporal: um estudo em crianças brasileiras. Psico (Porto Alegre), 41(4), 510-516.

Santini, A. P. \& Kirsten, V. R. (2012). Relação entre o perfil nutricional e a imagem corporal de escolares e adolescentes matriculados em escolas do meio rural da cidade de Santa Maria, RS. Revista da AMRIGS, 56(1), 32-37.

Santos, M. L. B., Monteiro, L. A. C., Silva, M. F., Sousa, M. S. C., \& Novaes, J. S. (2009). Imagem corporal e níveis de insatisfação em adolescentes na pós-menarca. Revista da Educação Física/UEM, 20(3), 333-341. https://doi. org/10.4025/reveducfis.v20i3.6830

Stice, E., Hayward, C., Cameron, R. P., Killen, J. D., \& Taylor, C. B. (2000). Body image and eating disturbances predict onset of depression among female adolescents: a longitudinal study. Journal of Abnormal Psychology, 109(3), 438-444. https://doi.org/10.1037/0021-843X.109.3.438

Seixas, C. M. (2009). Comer, demandar, desejar: Considerações psicanaliticas sobre o corpo e o objeto na Obesidade (dissertação). Rio de Janeiro (RJ): Instituto de Medicina Social da Universidade do Estado do Rio de Janeiro, Programa de pós-graduação em Saúde Coletiva.

Tabak, I., Mazur, J., Oblacińska, A., \& Jodkowska, M. (2007). Body mass, self-esteem and life satisfaction in adolescents aged 13-15 years. Medycyna wieku rozwojowego, 11(3), 281-290.

Triches, R. M, \& Giugliani, E. R. J. (2007). Insatisfação corporal em escolares de dois municípios da região Sul do Brasil. Revista de Nutrição, 20(2), 119-128. https://doi.org/10.1590/S1415-52732007000200001

Toni, V., Gavineski, I. C., Migon, P., Finato, S., Rech, R. R., \& Halpern, R. (2012). Insatisfação com a Imagem Corporal em adolescentes de Escolas Públicas de Caxias do Sul-RS. Revista Brasileira de Ciências da Saúde, 16(2), $187-194$. https://doi.org/10.4034/RBCS.2012.16.02.10

Vilela, J. E. M., Lamounier, J. A., Dellaretti-Filho, M. A., Barros-Neto, J. R., \& Horta, G. M. (2004). Transtornos alimentares em escolares. Jornal de Pediatria, 80(1), 49-54. https://doi.org/10.2223/1133

Welch, C., Gross, S. M., Bronner, Y., Dewberry-Moore, N., \& Paige, D. M. (2004). Discrepancies in body image perception among fourth-grade public school children from urban, suburban, and rural Maryland. Journal of the American Dietetic Association, 104(7), 1080-1085. https://doi.org/10.1016/j.jada.2004.04.019

Wiederman, M. W., \& Pryor, T. L. (2000). Body dissatisfaction, bulimia, and depression among women: the mediating role of drive for thinness. International Journal of Eating Disorders, 27, 90-95. https://doi.org/10.1002/(SICI)1098108X(200001)27:1<90::AID-EAT10>3.0.CO;2-0

Witt, J. S. G. Z, \& Schneider, A. P. (2011). Nutrição Estética: valorização do corpo e da beleza através do cuidado nutricional. Ciência \& Saúde Coletiva, 16 (9), 3909-3916. https://doi.org/10.1590/S1413-81232011001000027

Dados dos autores:

Miria Suzana Burgos - Doutora, Universidade de Santa Cruz do Sul.

Luciana Tornquist - Doutoranda, Universidade Federal de Pelotas.

Debora Tornquist - Doutoranda, Universidade Federal de Pelotas.

Edna Linhares Garcia - Doutora, Universidade de Santa Cruz do Sul.

Cézane Priscila Reuter - Doutora, Universidade de Santa Cruz do Sul.

Endereço para correspondência:

Luciana Tornquist

Rua Padre Anchieta, 4715, apto. 401, bloco E

96015-420 Pelotas, RS, Brasil

$<$ luciana.tornquist@yahoo.com.br>

Recebido em: 30.11 .2016

Aceito em: 03.01.2018 